\title{
Predicting a User's Numeric Identity from the Search of Attribute Data
}

\author{
Jacob MBAYDAY \\ Department of Mathematics and Computer Science \\ University of Ngaoundéré \\ Ngaoundéré, Cameroon
}

\author{
Paul DAYANG \\ Department of Mathematics and Computer Science \\ University of Ngaoundéré \\ Ngaoundéré, Cameroon
}

\begin{abstract}
In common Internet environments, most of the websites or services constrain the user account creation. Since the Internet is accessible by all and offers more and more services, a user has several accounts on the web. The difficulty in controlling their accounts does not leave indifferent to the users of the web. Hence the use of easy or insecure passwords. This is why we are victims of attacks and forgetting our passwords. Large companies such as Facebook, Google, etc., offer authorization and authentication mechanisms using the Oauth and OpenID protocol, which requires the opening of an account. To be independent of a social network or a site, it would be important to develop a model to make a statistical analysis between the attributes of the profiles of the same user and to create an account.

Using the same password for all its different accounts could be an approach but avoiding the proliferation of data by proposing a model of identity analysis would be even more interesting. That is why this article proposes a centralized account management model by making a comparative and statistical study of the identity attributes and proposing a single account to the user to manage all its different accounts. So, we have a horizontal analysis between the attributes of the identity categories and a vertical analysis between these categories. This study allowed us to find a threshold to conclude that an account belongs to a user.
\end{abstract}

Keywords: Identity; Numerical Identity; Attribute; OpenID; Oauth.

\section{INTRODUCTION}

With the emergence of the web in the early 1990s, websites grow exponentially. It thus appears that the existence of several sites with user accounts, in order to uniquely identify a user, implies the multiplicity of user accounts. There are thus some problems such as the management of the passwords; the lack of flexibility associated with the use of different combinations of username and password. We must find a solution to minimize the creation of accounts on the web by ensuring the portability of the identity of a user from one service to another in a structure to avoid the proliferation of certain attribute data.

In a structure, each service has its database and access to a service requires the existence of an account. This article, entitled "Contribution to the Prediction of a User's numeric Identity from the Searching of Attribute Data", thus demonstrates that it is possible to have a single account on the web, Access to any other service on the web. In most cases, the private structures have several services requiring the user to have an account for each of them. This results in duplication of user information and loss of time. In view of all this, it is essential to allow a structure to propose the connection to any service from a single account. To succeed in this challenge, it is a question for us to solve the problem of portability of information. That is to say how to allow a user to carry his data from one service to another.

The main objective of this article is to propose, from the search of attribute data, a model for predicting the numerical identity of a user in order to simplify the management of user accounts.

To achieve this, we need to deepen our knowledge of the concept of numerical identity and, on the other hand, to gather the needs for which we propose a solution that will be modeled according to the choices made.

\section{DATA MINING AND NUMERICAL IDENTITY}

\subsection{Numerical Identity}

The term "numerical identity" was chosen to make the link between real entity and virtual entity.

\section{Real identity}

To define the numerical identity, it is first necessary to distinguish the real world from the abstract people of the information systems [1]. For Roger Clarke [2]: "An identity exists in the real world and not on hard drives. It is a presentation or role of an underlying entity ". For him, an entity may represent a natural person, a legal person or an object. An entity may have multiple identities, depending on the role it plays. For example, a person may possess an identity associated with his or her professional role as an employee and a more personal identity as a relative in the household [1].

\section{Online identity}

In an information system, and particularly on social networks, an individual is often represented by so-called avatars. These avatars allow, for example, to contribute to an online journal or to bid on the Internet. The identity of these avatars, that is, their presentation or role, is often called a numerical identity. In view of the definition set forth above, this so-called 
numerical identity actually represents the identity of the avatar entity. The Anglo-Saxon definition speaks in this case of an online identity or identity on the Internet and its stakes are above all sociological. In [3], the authors propose a classification of this identity on-line by following the factors of the usual authentication technologies [7]. Figure 1 shows the two types of identities.

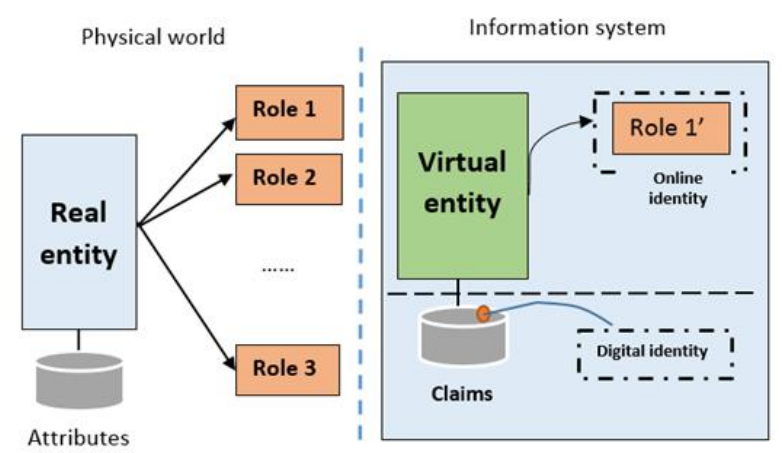

Figure 1: Representation of identity types (source: translated from [1]).

\section{Numerical Identity}

Like the identities of the real world, the identities of avatars possess a certain number of attributes which are represented in the information systems by recordings. As in the real world, some of these records allow an observer to distinguish between two identities. In the literature, numerical identity is considered as a set of records. In his laws of identity [8], Kim Cameron extends the notion of attributes for an identity by introducing the notion of "claims" within which the attributes are included. In particular, these claims are used to express the derivation of attributes such as age from the date of birth attribute. Cameron gives the following definition to numerical identity: a set of claims made by a numerical subject about himself or another numerical subject.

We define the numerical identity of a user as a global set of attributes that make up an on-line representation of who and what is the entity. It may include access credentials, personal attributes, and personal references. On the Internet, a user has many access qualifications that are published in different sites and different or multiple attributes and personal references on each site.

In each site, a user can be represented by subsets of these attributes. Depending on the situation and context, different subsets of attributes are used to represent the same user on the Internet. For example, in a bid site, a subset of a user's attributes such as user name, password and purchase history represent the identity of the user in this site, while a subset of the user's attributes such as the student ID number, the class record, can represent the identity of the user in a university site [4].
Numerical identity must be understood as self-image, selfexpression, which now incorporates all the behaviors, all the uses of numerical that are recorded. Numerical identity (IDN) can be defined as a technological link between a real entity (person, organization or company) and virtual entities (its numerical representations) [5].

Linguists would like to define the numerical identity as the form of the subject of online enunciation, that is, its pseudo, its avatar, its signature. The numerical identity is the set of personal data that we deposit or leave without our knowledge on the web, that is to say what we do, say, share, love, hate, seek, etc. This is the set of our numerical footprints, brands of our online presence. A like, a site registration, a book order or a ticket reservation, a payment by card, publication of photos are all elements of our online presence.

\subsubsection{Components and models}

\subsubsection{Components of the digital identity}

The digital identity of an individual is composed of formal data and informal. All these pieces of information make up a more global digital identity that characterizes an individual, his personality, his surroundings and his habits. These small pieces of identity function like genes: they compose the digital DNA of an individual [6].

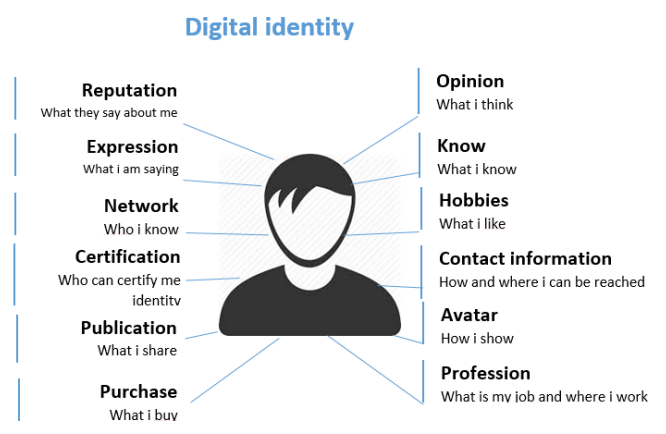

Figure 2: Digital Identity (source: translated from [6]).

\subsubsection{Identity system: form and model of the digital identity}

The development and evolution of the means of communication, through the multiplication of blogs and social networks, change the relationship between the individual and others. Thus, digital identity allows the identification of the individual online and the linking of the latter with this set of virtual communities that is the Internet. Hence, digital identity can be divided into three categories:

\section{The declarative identity}

The declarative identity refers to the data entered by the user as his name, date of birth, or other personal information directly informed by the individual. The information that 
makes up the declarative identity describes the person and makes it possible to distinguish it within the community [9].

\section{The acting identity}

Traces of user activity complete the identity structure. That is, the acting identity is the identity indirectly informed by the activities of the user on the web. They are derived from its deliberate interaction with the application: these are the friends, the objects gleaned during the practice of the software [9]

\section{The calculated identity}

The calculated identity results from an analysis of the identity acting by the system, such as the number of virtual communities in which the individual evolves or the number of friends on social networks. Unlike the declarative identity, the calculated identity is not filled by the user; unlike active identity, it is not the immediate product of its activity.

The discrepancy or at least the discrepancies that may exist between the declarative identity and the acting identity raise a major question. Who is really the individual we are dealing with on the web? [9].

By quantifying the presence, the visibility, the notoriety of the user, the calculated identity makes comparisons more or less explicit; It develops a disproportionate importance of the figure in the identity system and reflects the actions of the user in the local cultural mirror, implicitly implying a form of social play. [10].

\subsubsection{Managing and portability of a user's identity}

\subsubsection{Digital Identity management}

Digital identity management is the combination of a set of processes and a technological infrastructure for the creation, maintenance and use of these identities [11]. Digital identity management therefore consists of several tasks such as maintaining user attributes and using subsets of attributes to enable secure online interactions between users or between users and systems. Digital identity management allows the addition, use, and deletion of identity attributes.

Identity management systems are categorized into three models: isolation management, centralized, and distributed identity. In the identity management isolation model, each site has its own identity management domain and its own way of maintaining user identities including employees, customers, and associates. The centralized identity management model has a simple identity provider to which brokers trust other participating members or service providers in a circle of trust. The Distributed Identity Management model provides a frictionless identity management solution by forming a federation and making authentication a distributed task. Each member agrees on trusted user identities guaranteed by other members of the federation.

\subsubsection{Probability of the digital identity}

The importance of the portability of the user's digital identity should be addressed in central user identity management procedures. In other words, users should be able to export their digital identities and transfer them to various computers in a secure manner

\section{Data Probability}

Data portability refers to the ability to manage personal data on its own, to bring it from one system to another, to share it among several systems.

\section{Login probability}

Systems like openID can only create a login / password and use it on multiple sites

\begin{tabular}{|c|c|c|c|c|}
\hline \multicolumn{2}{|c|}{ Typology } & \multicolumn{3}{|c|}{ Examples } \\
\hline 总 & Shifters & Anarchy online & $M S N$ & Livejournal \\
\hline 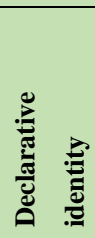 & Qualifiers & $\begin{array}{l}\text { Character search, } \\
\text { craft, function }\end{array}$ & $\begin{array}{l}\text { Profile } \\
\text { search, } \\
\text { currency, } \\
\text { Interests }\end{array}$ & $\begin{array}{l}\text { Personal } \\
\text { information } \\
\text { biography, } \\
\text { interests }\end{array}$ \\
\hline & Action & $\begin{array}{l}\text { Pick up an object, } \\
\text { cast a spell }\end{array}$ & $\begin{array}{l}\text { Discuss, } \\
\text { modify }\end{array}$ & $\begin{array}{l}\text { Publish, } \\
\text { comment }\end{array}$ \\
\hline$\stackrel{\theta 0}{\Xi}$ & Community & Guild, group & Friends & Favorites \\
\hline 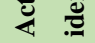 & Collection & Inventory items & & \\
\hline 营 & Numbers & $\begin{array}{l}\text { Number of } \\
\text { teammates, hit } \\
\text { points, experience } \\
\text { points }\end{array}$ & $\begin{array}{l}\text { Number of } \\
\text { friends } \\
\text { connected }\end{array}$ & $\begin{array}{l}\text { Number of } \\
\text { comments, } \\
\text { visits, login } \\
\text { date }\end{array}$ \\
\hline
\end{tabular}

Table 1: Examples (instant messaging, blog)

\section{Probability of identity}

On many websites, you can create your profile. A user who uses a large number of websites must do so each time and, in general, enter the same information (surname, first name, address, etc.). Technically, the hCard microformat allows to expose its profile data

\section{Probability of social networks}

For Internet users who are members of several social networks, maintaining links with friends within these networks is a heavy business. The elementary data here is the 
information that the person $\mathrm{X}$ is a contact of the person $\mathrm{Y}$. The objective of data portability is to be able to export these links between people from one site to another or to manage them outside of any proprietary website.

\section{Probability of interests}

The user, in his interaction with an online service, often declares data that are related to his "attention", ie to his interests. In addition to these declarative data, a certain number of data are also automatically deduced from its behavior (page views, clicked keywords, purchases, etc.).

\section{Authorization management}

For some luxury cars, there are special keys that you can give to a valet and that do not allow you to drive for more than a few kilometers, or do not give access to the GPS (Global Positioning System) or to the address book of the car. Phone. The idea of OAuth is in the same spirit: to give access to a third party to a part of its data only [12].

\subsection{Data mining}

\subsubsection{Definition and types of data}

Data mining can be defined as "a process of non-trivial extraction of implicit information previously unknown and potentially useful from data from the database". It consists of searching for and retrieving large quantities of data stored in databases or data warehouses (useful and unknown) [13].

Data mining factors are:

- A large computing powers

- The basic volume of data increases enormously

- $\quad$ The access to the global network

- Awareness of the commercial Internet to the optimization of manufacturing processes, sales, management, logistics

The data types are [13].

- Discrete data: binary data (sex ...), enumerative data (color ...), and ordered enumerations (answer 1: very satisfied, 2 : satisfied...).

- Continuous data: whole or real data (age, salary...).

- Dates

- Textual data

- $\quad$ Pages / web links, Multimedia.

\subsubsection{Data mining: Motivation}

In [14], data mining is motivated by the following arguments:

- The process data concern both qualitative and quantitative attributes, which justifies the of discretization steps to obtain Boolean contexts
- The data are voluminous: Items per million, attributes thousands. These characteristics pose many algorithmic problems

- The data mining pursues a goal of exhaustiveness discovered knowledge. At the difference statistical techniques, that are not only global trends of the data that are sought but also local properties that concern a small number of objects

- In the light of the exploration methods used to assist the expert in his decision-making, it is desirable that the assistance provided be clearly justified, explained and understood

The exponential increase in the number of Internet users gives rise to what is now called the explosion of data [14].

- Large mass of data (million billion bodies): it doubles every 20 months

- Data are multidimensional (thousands of attributes

- Unusable by conventional methods of analysis

- Collection of large masses of data (Gbytes / hour)

- $\quad$ Satellite data, genomics (microarrays, ...), scientific simulations, etc

- $\quad$ Need for real-time processing of this data

\section{DIGITAL IDENTITY MANAGEMENT SYSTEM}

\subsection{Identity management models}

Identity Management Systems (IMS) can be categorized into three types. Type 1 and Type 3 IMSs are intended to allow the use of a digital identity to establish a trust relationship while Type 2 IMSs are intended to monitor the use of a digital identity. Their classification refers to the work of Jøsang [15].

\subsubsection{Isolated management: Single database for each service}

\subsubsection{Definition and advantage}

This is the historical model for managing identities on a system. Each different system will act as both a service provider and an identity provider by implementing a database of its users. The classic use case of an identity in this model is the authentication of the user requesting a service. In this model, the service provider has the list of identities and itself implements the necessary authentication functions. It is therefore in charge of both identity and service management. It is also up to him to ensure the security and protection of privacy.

\subsubsection{Centralized management: common user}




\subsubsection{Definition and advantage}

We sought to centralize identities in one and the same identity provider to remedy the problem of the multiplicity of identities. For this purpose, the first method is to provide a common identifier for all services. In this model, a unique identity provided by an identity provider is used to authenticate a user to several service providers in the same security domain.

In RFC 4513 [16], it is specified that messages between the server and the client must be encrypted using the TLS protocol [17] to ensure confidentiality and integrity of the exchanges. The use of directories has greatly simplified the management of identities, especially in the corporate world. However, in an open world like the Internet, it is impossible to offer a global directory. This is why the other methods have been proposed to manage digital identities in order to allow for a scaling-up of the architecture.

The use of IGC is now almost systematic for non-human entities (server, websites). This is the case, for example, when establishing secure connections with Transport Layer Security (TLS) [17] on websites. Today, most sites that have a private part is accessible in HTTPS and has a certificate signed by a recognized authority of the browser [1].

\subsubsection{The limits}

Whenever possible, mutual authentication using certificates is only scarcely widespread due to the difficulty of managing certificates by individuals. The use of certificates also poses a problem of privacy. Indeed, certificates are easily traceable, the link with the entity they designate is often obvious and hiding information is not possible. Certificates also do not allow expressing complex claims and are limited to expressing attributes.

\subsubsection{Meta-identity management model}

Service providers can share a number of identities needs that can be grouped under a meta-identity. In this model, shown in FIG. 7, the identities of the subject are linked to a meta-entity that will be used to access services present in a security domain. Identity is provided by a single identity provider that is responsible for authentication and therefore allows single sign-on mechanisms (SSO)
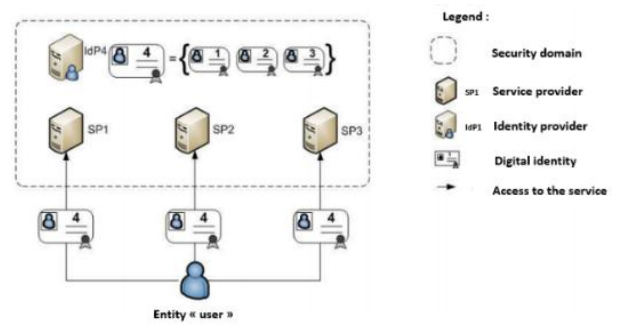

Figure 3: Management model with a meta-identity (source: translated from [1]).
This model is present in large companies where services are controlled and where there are equivalent security policies for all service providers. This model is not applicable to an open environment where precisely the policies and security requirements differ from one supplier to another. Moreover, the use of a meta-identity is a risk to the privacy of the users since the latter contains several of the original identities

\subsubsection{Federated identity management}

Camenisch and Pftizmann [18] introduce the notion of federation of identity allowing the sharing of numerical identities between several security domains within a circle of confidence. A trusted circle consists of several service providers who will trust one or more identity providers to authenticate users. The goal of this federation is to allow users to access the system from another security domain without the need to change their identity.

\subsubsection{User-centric management}

An identity management solution must take into account how the user will use his or her identity. The models presented above are mainly used to facilitate identity management for service providers, with the exception of identity federation intended to simplify user use. However, the federation model is not realistic on a large scale because it is impossible to create a global federation. This is why the current vision of identity management is to help the user manage his identity directly while maintaining strong constraints on security.

\subsubsection{The online model}

The principle of user-centric identity management is to allow the user to more precisely control his identity and to place himself at the center of identity exchanges. The model we have described as "online" assumes that management does not require any user-side adjustments and that it will allow it to manage its identities in its identity provider (s) directly from a browser. Examples of this type of identity management can be found in the OpenID and OAuth protocols.

\subsubsection{The smart client model}

This model assumes that the user who seeks to use his identity with a service provider has a tool (the client) to enable him to manage his identity. Implementations of this model focus on the problem of privacy by offering a direct control by the user. Many of these clients are integrated directly with browsers. These customers are mostly local password managers who only address the issue of user use

\subsubsection{The Identity management meta- system model}

The model of identity management meta-system defined in [4] proposes an abstraction layer based on the seven (7) laws of identity stated in [19]

- User control and consent

- Minimum disclosure for a defined use

- Legitimate Parties: The user must be notified of the parties transmitted their personal information 
- Directed Identity: An identity management system must manage both omnidirectional identifiers used by public entities and unidirectional identifiers used for private purposes

- Pluralism of Operators and Technologies

- Human Integration: A Meta identity system must put the human user as a system component

- Consistent experience between contexts: A Meta system must ensure a consistent user experience while retaining the plurality of identity management systems

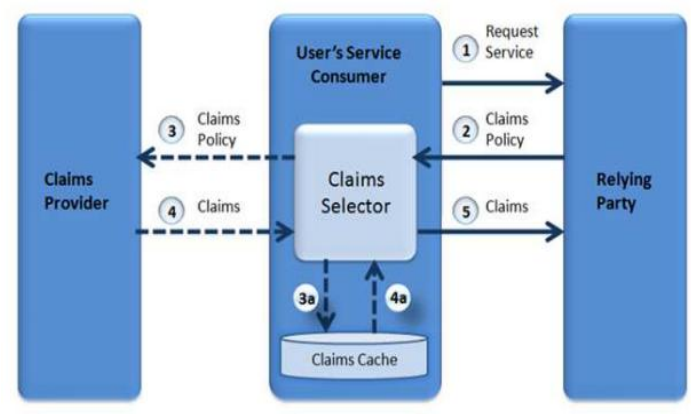

Figure 4: The identity meta-system (source: [1])

The main element of the system is the Meta identity selector that allows the user to choose the identity he wants. The identity selector works as a portfolio in which we possess several business cards that could be used to prove identity.

\subsection{Protocols and languages for identity management}

The models that we have described are theoretical models of identity management. To allow the implementation of complex models that are running user-centric and identity federation for example, a number of protocols and specific languages have been proposed.

\subsubsection{OpenID}

The open standard OpenID [20] was originally developed for the site LiveJournal. This protocol is based on HTTP protocols [21] and HTTPS [22] and the use URI or XRI [23] as identifiers. It also uses signature algorithms HMAC-SHA1 [24] and (hash-based message authentication code - Secure Hash Algorithm 256) HMAC-SHA256 [25] and performs discovery of the IdP from an XML document. It has boomed because of its simplicity and the important role given to the user. The latter has full freedom to choose his identity provider and may even become his own supplier. It also controls the data passed to service providers. Basic operation is as follows:

- The user initiates authentication with an ID (URL or XRI) in the login field of the site (Relying Party).

- The identifier is then provided "parsed" in order to know the URL to the OpenID Provider accepts messages.

- Optional step : The Relying Party and Open ID provider to agree on a shared secret. This secret will be used to check the following messages between the OpenID Provider and Relying Party.

- The user is redirected by the Relying Party to its Open Provider ID with an authentication request.
- Open ID provider verifies that the user is authorized to make this authentication.

- Open ID provider redirects the user to the Relying Party indicating whether the authentication is successful.

-Relying party verifies the information received, either by using the negotiated key 3 is directly by requesting the Open ID Provider.

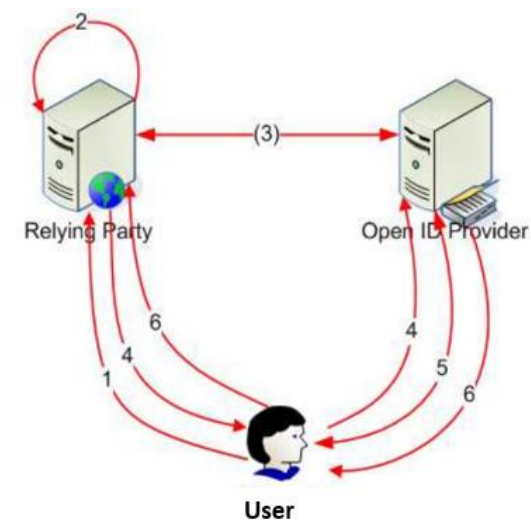

Figure 5: Schematic flow OpenID

\section{The limits of OpenID}

Several limitations are known on using OpenID: the first is that the security authentication is left to the OpenID Provider (step 5); the second is that the OpenID system is vulnerable to phishing. In fact, a fraudulent site can lure the user into believing that he is redirected to his Provider (step 4) to the login phase and can then present a fake login form to collect the username and the password of the latter.

OpenID responds initially to the question of simplicity. The solution also meets the problems of respect for private life by clearly people which data are exchanged. For cons, the identity security aspect is left to the developer for the whole party authentication and storage of identity.

\subsubsection{Oauth}

The OAuth protocol [25] was proposed in 2006, allows for the delegation of access to an API securely. This protocol specifically addresses access to resources authorizations. OAuth follows the model online; it is based on protocols such as HTTP [21] and HTTPS [22] and the use of signature algorithms like HMAC-SHA1 [24] and RSA-SHA1 [26]. The classic scenario of the protocol is secure use and approved by the protected resource user in a service provider from a site called consumer. The sequence of steps of the protocol is as follows:

- User visits a consumer website in which he will want to use another site resources.

- The consumer website contact the service provider to obtain a temporary debt in order to identify the delegation request.

- The valid query service provider and consumer website responds to a set of temporary loans.

- The consumer site then redirects the user to the service provider to obtain the approval of the user using temporary loans previously received. 
- The service provider then authenticates the user with a method of their choice and request consent to the user to share its resources with the consumer site.

- The service provider redirects the user and informs the consumer that the user has consented to the exchange.

- The consumer site then requires a set of claims for the application of resources from the service provider. The request is made using temporary receivables and through a secure channel with TLS.

- The service provider returns the set of claims.

- Use of these receivables, the consumer can make the request of the service provider's resources.

\section{The limits of OAuth}

The protocol ensures message integrity, but does not address the issue of confidentiality of trade. It is recommended to use it over a secure connection. Similarly, the authenticity of the service providers is not verified which therefore creates a risk of usurpation of the identity of these and also makes it sensitive protocol to phishing attacks. In this case, the user enters his login information in a fraudulent service provider. By requiring the consent of the user, OAuth addresses the issue of protection of privacy, however, the protocol does not ensure compliance with the other assumptions for the protection of the latter and the limitations in terms of confidentiality still pose here problem

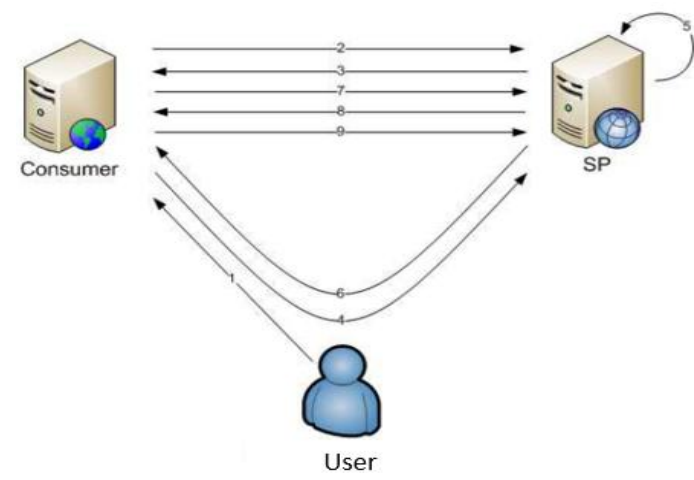

Figure 6: Schematic flow OAuth

\subsubsection{SAML2}

The Security Assertion Markup Language 2.0 (SAML2) is a standard proposed by OASIS (Organization for the Advancement of Structured Information Standards) dedicated to exchanging authentication and authorization data between security domains. In version 2 , this standard has integrated the work of the Liberty Alliance consortium on ID-FF framework [27] to allow the federation of digital identities. SAML2 defines the syntax of identity assertions encoded with XML. [28] These can be signed using XML signature mechanisms [29] in order to certify the content. These assertions may correspond to authentication data, authorization or just the attributes of an entity. SAML2 defines a number of protocols for the treatment of requests and replies using these assertions. Similarly, assertions are usually transported to other structures such as HTTP POST requests or SOAP messages. The standard therefore defines methods to achieve this link and transport SAML2 assertions. Finally, the standard OASIS offers a number of profiles that match different use cases including SSO and identity federation. The SAML2 standard can be used in different identity management models and is not tied to a single type of model.

\section{The limits of SAML2}

SAML was never designed to enable the SSO to the new generation of native mobile apps, or to those that consolidate data and services, from different sources, through calls to APIs. WS-Trust (for SOAP services), OAuth 2.0, an open standard used for authorizations, and OpenID Connect, which is based on a specification of OAuth, were designed to address these issues and bring users more value and flexibility. Some organizations may choose to use SAML without direct LDAP connection. Although this configuration is possible, some IBM Maximo Asset Management functions require a direct connection to function properly.

\subsection{Connection APIs}

APIs (Application Programming Interface) interfaces are essential for inter-application exchanges, but also a philosophy of openness of its information system architecture and operational implementation. The management API that develops, also includes safety and supervision, to name a few. The APIs are a way to equip the digital transformation, to go from digital strategy to implementation.

When you connect to a site using the Google or Twitter account is a Google API or Twitter that can benefit the other site of the service ... and the way to recover your data on Google and Twitter.

When mobile of our favorite newspaper shows us the weather is certainly an API that is action. And perhaps it was marketed to this news site, weather data company that sells its data in the form of services.

The APIs are at the heart of the evolution of commodity Internet and new ecosystems. Perhaps the future business model of our company. Some "Chief Digital Officer" more advanced than others, because their industry is more exposed to the digital transformation, have already understood the stakes APIs and also structure of internal initiatives around IT architecture.

Hence the idea of trading in these new ecosystems around APIs, API during monthly gatherings Connection, and to link the actors in all industries, trades with the geeks, the ideas with tracks solutions and exchange around the initial feedback. [30].

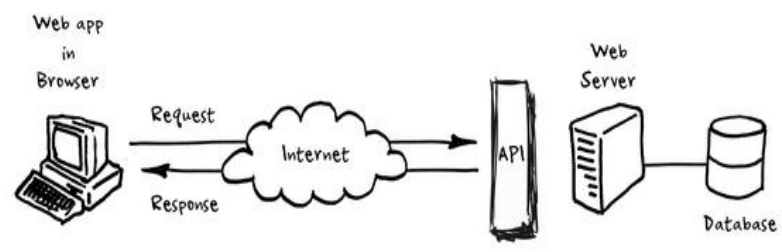

Figure 7: Connection API (source: [30])

\section{PROPOSAL AND MODELING SOLUTION}

In this interconnected world, the need not identify themselves only once for several sites or gadgets and specially to have fewer attributes on the Internet has become indispensable and a new term "digital identity" was born. Unfortunately, this 
need has not been identified during the creation of the first protocols underlying the Internet. Therefore, backup solutions were more or less successfully developed and led to an explosion of methods and tools for managing identities.

\subsection{Proposed solution}

Currently, identity management models are booming and the true face of the user to be determined. We therefore propose to determine this face through this study: contribution to the prediction of the digital identity of a user from the attribute data mining. We will, from the declarative and operative identity, determined who is really the person to whom we have to do on the canvas. It is therefore necessary to collect the attributes of these identities and making a horizontal analysis between entities and vertical between identity categories. We also believe that instead of using OpenID authentication or authorization with OAuth, you must do a statistical analysis of the accounts of a user and find a unique path to its accounts.

\subsection{Modeling of the solution}

\subsubsection{Interactions between the three categories of identity}

The identity system as a whole is shown in the diagram below.

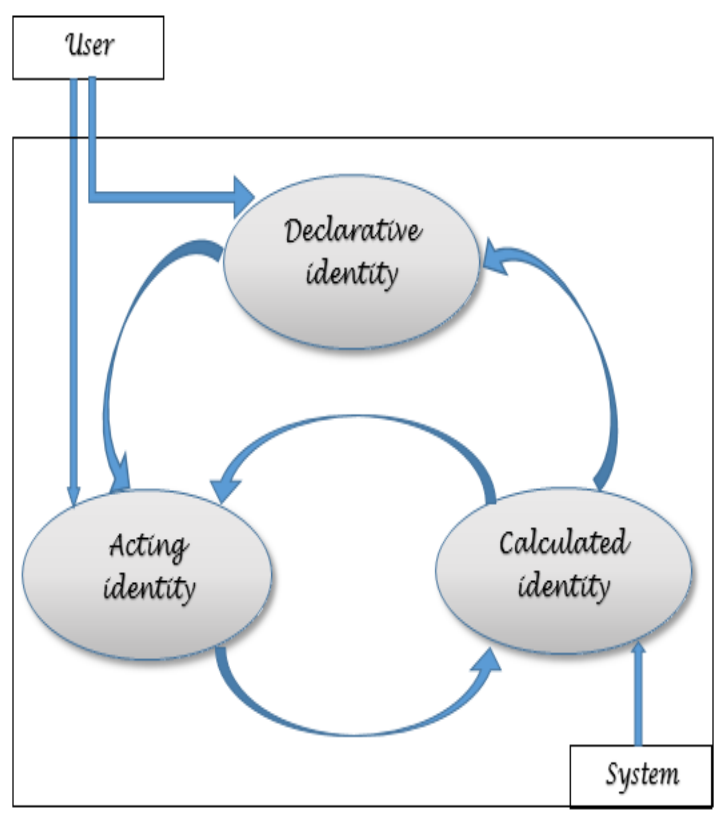

Figure 8: identity system as a whole

-User: one that is central to any identity. He leaves enormous information during the creation of account and especially when the use of his account.

- Declarative identity: User information such as name, first name, date of birth, country, profile picture, etc. provided directly by the user will give rise to the declarative identity.

-Acting identity: requesting friends, participating in an event or group, commenting or tagging or sending a gift, discussing, posting, commenting, etc. It is the product of the user and of the declarative identity without forgetting a slight participation of the identity calculated computing.
- Calculated identity: it depends on the acting identity and especially on the system. By using data of the acting identity, the system makes calculations to evaluate the activities of the user in number.

\subsubsection{Identity system end data backup}

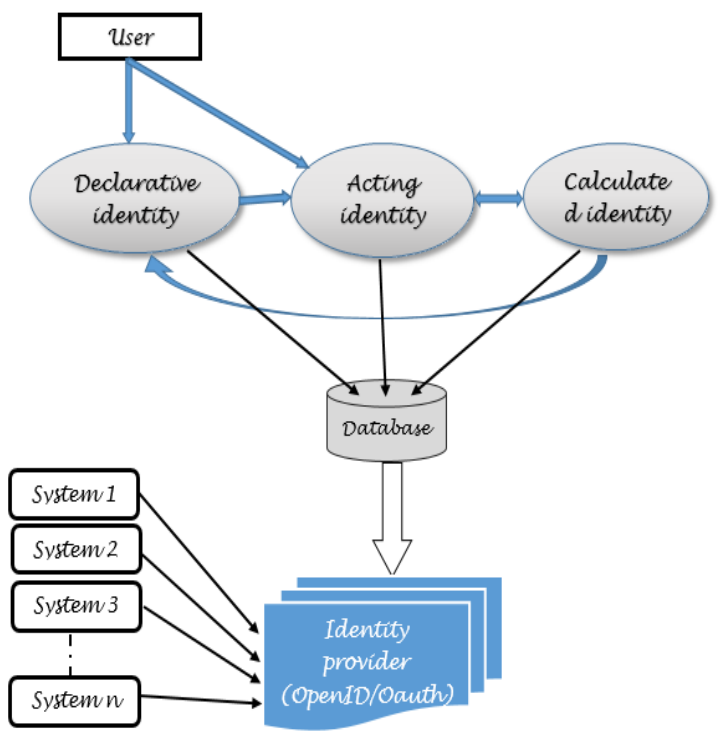

Figure 9: Identity System and data backup

All components of declarative identities, active and calculated will be saved in a database. Thereafter the data will be redirected to an OpenID or OAuth server for the unique and complete identity. This server must be accessible by any system requiring the user data.

\subsubsection{Horizontal analysis between data}

All data of each model are collected in a table and are listed in order of appearance when creating an account. The stability of a component shows more clearly that it is an important attribute in relation to that which varies regularly. The stability is determined over time. The system will therefore be based on the time that is an attribute since its appearance until analysis.

Non exhaustive list of some information collected from social networks like Facebook, twitter, 
International Journal of Computer Applications Technology and Research

Volume 10-Issue 06, 166-177, 2021, ISSN:-2319-8656

\begin{tabular}{|c|c|c|}
\hline $\begin{array}{l}\text { Declarative } \\
\text { identity }\end{array}$ & Active identity & Calculated identity \\
\hline - Name & - friends request & - number of friends \\
\hline - Profile Sheet & - send Tweets & - number of groups \\
\hline $\begin{array}{l}\text { - Avatar / } \\
\text { photography }\end{array}$ & - sent a joint post & - number of events \\
\hline - Race & $\begin{array}{l}\text { - has been tagged by a } \\
\text { friend }\end{array}$ & $\begin{array}{l}\text { - date of the last } \\
\text { connection }\end{array}$ \\
\hline - Function & - discuss & - number of comments \\
\hline - Gender & - publish a text & - number of visits \\
\hline - Birth date & - Comment & - number of positions \\
\hline - country & - updated profile & $\begin{array}{l}\text { - number of } \\
\text { teammates }\end{array}$ \\
\hline - The interests & $\begin{array}{l}\text { - used an X } \\
\text { application }\end{array}$ & $\begin{array}{l}\text { - subscriptions } \\
\text { Volumes }\end{array}$ \\
\hline $\begin{array}{l}\text { - Personal } \\
\text { informations }\end{array}$ & $\begin{array}{l}\text { - Volume of sent } \\
\text { tweets }\end{array}$ & - attendance rate \\
\hline$-\ldots$ & $-\ldots$ & $-\ldots$ \\
\hline
\end{tabular}

\begin{tabular}{|c|c|c|c|}
\hline \multirow[b]{2}{*}{ Order } & \multicolumn{3}{|l|}{ Index } \\
\hline & $\begin{array}{l}\text { declarative } \\
\text { identity }\end{array}$ & active identity & calculated identity \\
\hline 1 & $\begin{array}{l}\text { Last name and } \\
\text { first name }\end{array}$ & Friends request & Number of friends \\
\hline 2 & Birth date & tweets sent & Number of groups \\
\hline 3 & Sex & $\begin{array}{l}\text { Participation in } \\
\text { events }\end{array}$ & number of events \\
\hline 4 & Race & Create Group & attendance rate \\
\hline 5 & Country & Update Profile & $\begin{array}{l}\text { number of } \\
\text { teammates }\end{array}$ \\
\hline 6 & $\begin{array}{l}\text { Avatar / } \\
\text { photography }\end{array}$ & $\begin{array}{l}\text { has been tagged by a } \\
\text { friend }\end{array}$ & $\begin{array}{l}\text { number of } \\
\text { comments }\end{array}$ \\
\hline 7 & Biography & used application & number of visits \\
\hline 8 & Pseudonym & $\begin{array}{l}\text { commented or } \\
\text { tagged }\end{array}$ & $\begin{array}{l}\text { Date of } \\
\text { connection }\end{array}$ \\
\hline 9 & Hobbies & $\begin{array}{l}\text { sent a collective } \\
\text { post }\end{array}$ & $\begin{array}{l}\text { Number of } \\
\text { position }\end{array}$ \\
\hline 10 & $\begin{array}{l}\text { Personal } \\
\text { informations }\end{array}$ & Discuss & $\begin{array}{l}\text { Volumes } \\
\text { subscriptions }\end{array}$ \\
\hline$\cdots$ & $\ldots$ & $\ldots$ & $\ldots$ \\
\hline
\end{tabular}

After collecting data from each category of identity we make a horizontal study between attributes and vertical study between identity categories. For this vertical study, the result of each pair of feature is stored in tables (T1 to ID and D-IDA, T2 for ID-A and ID-C, T3 for ID-ID and D-C). We are a horizontal analysis between these tables for a final identity. After getting a true identity of a user based social networks or websites we propose a system with all necessary attributes for enabling a user to identify himself once for all other existing systems on the canvas.

The horizontal study is to classify entities of these identities in order of relevance, importance and especially stability over time. The information characterizing the precise and safe way users are most important.

Table 3: Study of horizontal attributes.

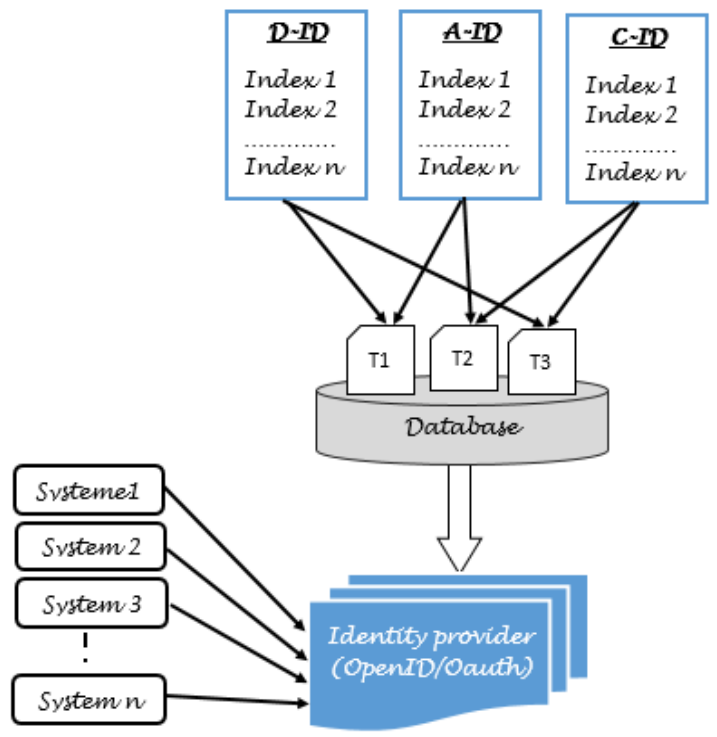

Figure 10: vertical and horizontal analysis of all types of identity 
The index order depends on the stability over time. T1 (T2 respectively T3) contains the D-ID attributes and A-ID (respectively A-ID and C-ID, D-ID and C-ID) in order of stability and reliability.

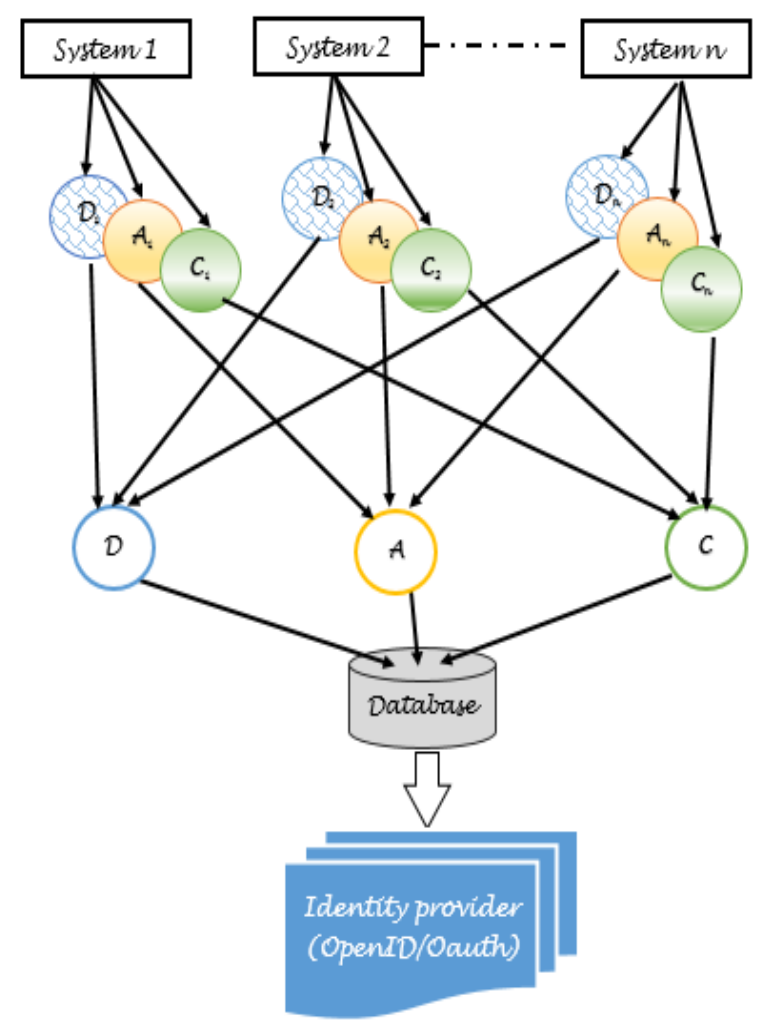

Figure 11: Multi-system

It is considered $\mathrm{n}$ systems each consisting of triplet (Dn, An, $\mathrm{Cn}$ ) identity categories. We collect all the declarative identity (respectively the acting identity and calculated identity) of each system with the connection API to form the unique identity declarative D (respectively A and calculated active C). We seek a threshold to conclude whether it can merge all these systems and find one user or whether these attributes cannot allow us to conclude. Vertical and horizontal studies will be made on the final product. If we find an important threshold, the data will be saved in a database and redirected to a service provider.

The systems do not have the same objectives it is not possible to have the same components in each category above the calculated identity and identity as the active identity. To make a comparison between the attributes of identities can be based on profiles, names, surnames, date of birth, country, gender etc. for declarative identities we rely on facial recognition and comparison of profiles at the names, sex, country and date of birth will be from one system to another.

\section{RESULTS AND DISCUSSION}

\subsection{Results}

\subsubsection{Collected attributes Couples}

Information was collected on 60 user profiles where each user has two accounts. We rely on attributes such as names, Name, Phone Number, Date of Birth, Sex, profile picture, personal information, interests center, Marital status and religious.

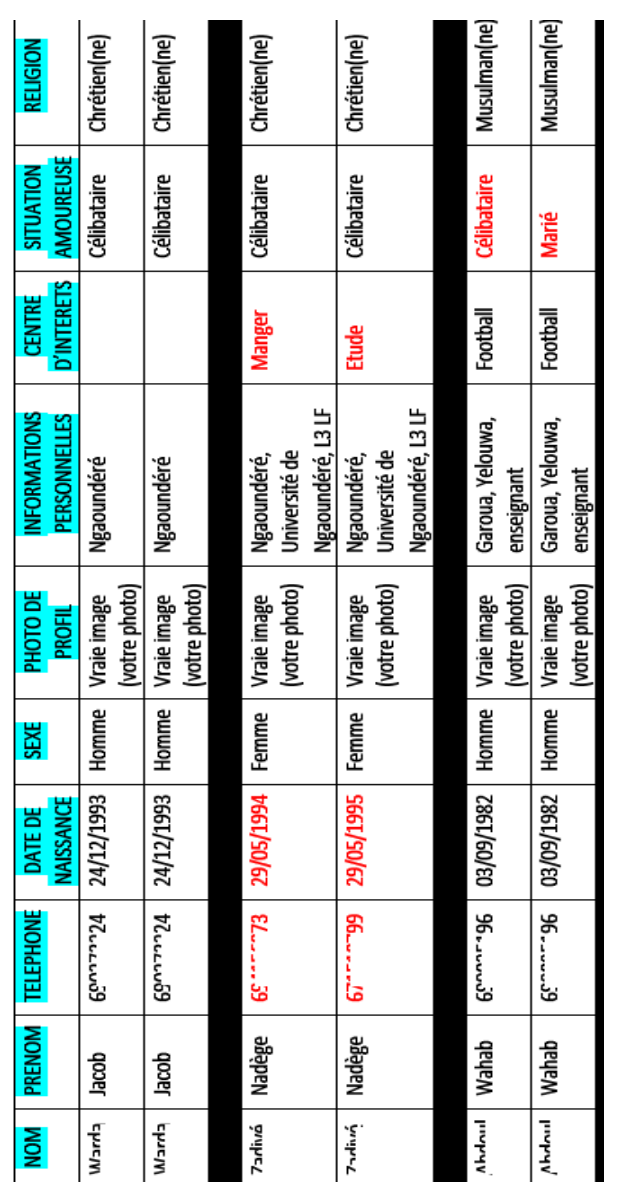

Table 3: presents the first 3 pairs collected accounts illustration.

\subsubsection{Analysis attributes collected}

After having collected the data we have red incompatible attributes couples and those black compatible. By focusing us on couples of compatible attributes we get the diagram expressing percent data reliability.

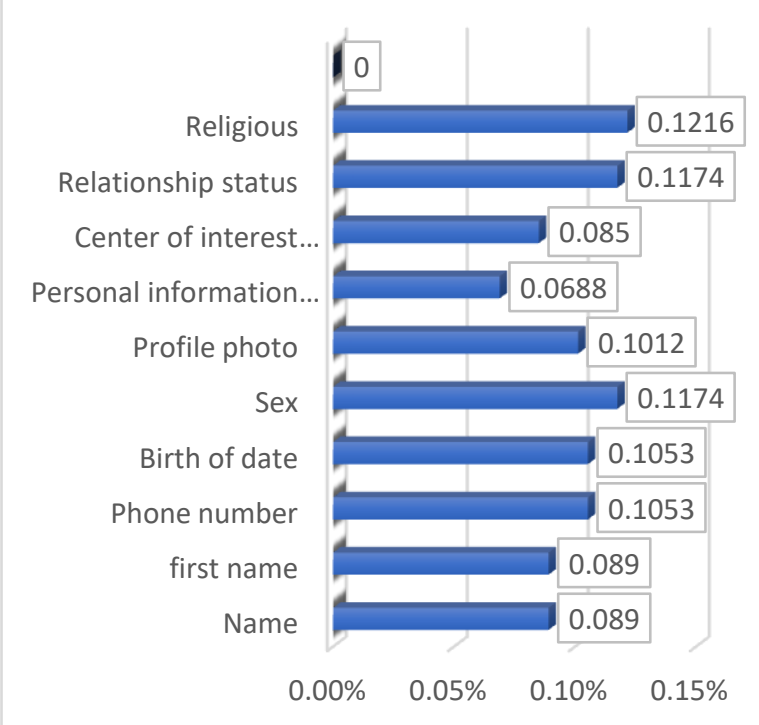

Diagram 1: data collected 


\subsubsection{Classification of attributes}

We classify our data into two categories: Fort weight and low weight. The choice of these categories is based on stability data for the most stable data are the most characteristic, if not the most telling. When creating an account entity such as Name, Date of birth and phone number are not easily changeable and the user think almost to the modification of such information. Profile photo on it is an element that is updated almost all the time and remains a very important tool to identify a user.

\begin{tabular}{|c|c|c|c|c|c|}
\hline \multicolumn{5}{|c|}{ Heavy weight } & \\
\hline $\begin{array}{l}\text { Dirth of } \\
\text { date }\end{array}$ & $\begin{array}{l}\text { Phone } \\
\text { number }\end{array}$ & $\begin{array}{l}\text { Profile } \\
\text { photo }\end{array}$ & Name & $\begin{array}{l}\text { First } \\
\text { name }\end{array}$ & Total \\
\hline $15.05 \%$ & $10.53 \%$ & $\begin{array}{l}10.53 \\
\%\end{array}$ & $\begin{array}{l}10.12 \\
\%\end{array}$ & $8.9 \%$ & $\begin{array}{l}48.98 \\
\%\end{array}$ \\
\hline
\end{tabular}

Table 5: Categorization: Heavy weight

As for the category of low weight, the choice is based on the instability and diversity of information such personal information and center of interest. It is also based on attributes in easy choice as sex, religion and relationship status.

\begin{tabular}{|l|l|l|l|l|l|}
\hline \multicolumn{7}{|c|}{ Low weight } & \multirow{2}{*}{$\begin{array}{l}\text { Total } \\
\text { Religio } \\
\text { us }\end{array}$} & Sex & $\begin{array}{l}\text { Relations } \\
\text { hip status }\end{array}$ & $\begin{array}{l}\text { Center of } \\
\text { interest }\end{array}$ & $\begin{array}{l}\text { Personal } \\
\text { information }\end{array}$ & $\begin{array}{l}51.02 \\
\%\end{array}$ \\
\hline $\begin{array}{l}12.16 \\
\%\end{array}$ & $\begin{array}{l}11.74 \\
\%\end{array}$ & $11.74 \%$ & $8.5 \%$ & $6.88 \%$ & \\
\hline
\end{tabular}

Table 6: Categorization: low weight

\subsubsection{The threshold}

The threshold will be defined according to two categories of attributes we have defined it above. The attributes of the most significant are the most important and therefore should be the threshold to consider. After a thorough study of our sample we find that the user who provided the information less compatible $62 \%$ compatible attributes between the two accounts. And to be more sure we are increasing our $70 \%$ threshold and we attribute this to the high weight percentage. The validation of the attributes of the most significant confirms the identity of a user. Having found $\mathrm{p}_{\mathrm{i}}$ and the threshold we apply the following formula to find the new percentage noted $\mathrm{p}_{\mathrm{i}}^{\prime}$ :

$$
\mathrm{p}^{\prime} \mathrm{i}=\frac{70 p i}{48.98}
$$

\begin{tabular}{|c|c|c|c|c|c|c|}
\hline & \multicolumn{5}{|c|}{ Heavy weight } & \multirow[b]{2}{*}{ Total } \\
\hline $\mathrm{i}$ & $\begin{array}{c}\text { Dirth } \\
\text { of date }\end{array}$ & $\begin{array}{l}\text { Phone } \\
\text { number }\end{array}$ & $\begin{array}{c}\text { Profile } \\
\text { photo }\end{array}$ & Name & $\begin{array}{l}\text { First } \\
\text { name }\end{array}$ & \\
\hline $\mathrm{p}_{\mathrm{i}}$ & $10.53 \%$ & $10.53 \%$ & $10.12 \%$ & $8.9 \%$ & $8.9 \%$ & $\begin{array}{l}48.98 \\
\%\end{array}$ \\
\hline $\mathrm{P}_{\mathrm{i}}^{\prime}$ & $15.05 \%$ & $15.05 \%$ & $14.46 \%$ & $\begin{array}{l}12.72 \\
\%\end{array}$ & $\begin{array}{l}12.72 \\
\%\end{array}$ & 70 \\
\hline
\end{tabular}

Table 7: Percentage of high order.

Low weight attributes occupy the remaining $30 \%$. To find the new percentage we apply the formula.

$$
\mathrm{p}^{\prime} \mathrm{i}=\frac{30 p i}{51.02}
$$

\begin{tabular}{|c|l|l|l|l|l|l|}
\cline { 2 - 6 } \multicolumn{1}{c|}{} & \multicolumn{5}{c|}{ Low weight } & \multirow{2}{*}{} \\
\hline $\mathrm{i}$ & $\begin{array}{l}\text { Relig } \\
\text { ious }\end{array}$ & Sex & $\begin{array}{l}\text { Relatio } \\
\text { nship } \\
\text { status }\end{array}$ & $\begin{array}{l}\text { Center } \\
\text { of } \\
\text { interest }\end{array}$ & $\begin{array}{l}\text { Personal } \\
\text { informati } \\
\text { on }\end{array}$ & Total \\
\hline $\mathrm{p}_{\mathrm{i}}$ & $\begin{array}{l}12.16 \\
\%\end{array}$ & $\begin{array}{l}11.74 \\
\%\end{array}$ & $\begin{array}{l}11.74 \\
\%\end{array}$ & $8.5 \%$ & $6.88 \%$ & $\begin{array}{l}51.02 \\
\%\end{array}$ \\
\hline $\mathrm{P}^{\prime}{ }_{\mathrm{i}}$ & $\begin{array}{l}7.15 \\
\%\end{array}$ & $6.90 \%$ & $6.90 \%$ & $5.00 \%$ & $4.05 \%$ & $30 \%$ \\
\hline
\end{tabular}

Table 8: Percentage of low weight.

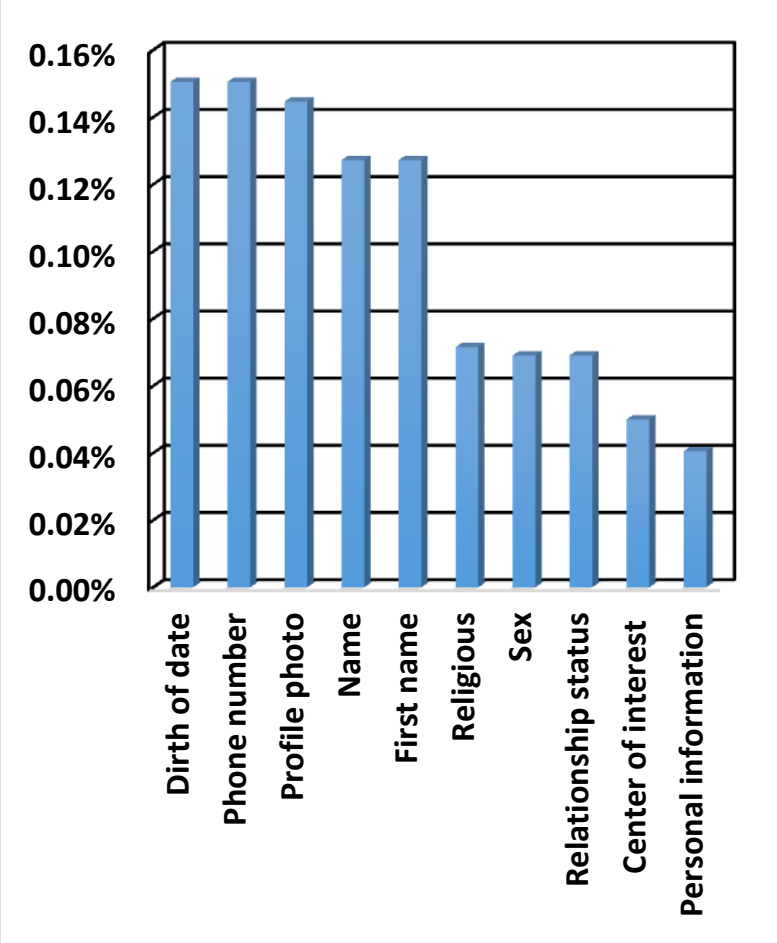

Diagram 2: Overview.

\subsection{Discussion}

Walking through the contours of digital identity, we find some digital identity management systems like OpenID that allows a user to authenticate to multiple sites without having to remember an identifier for each of them but each time using a unique identifier OpenID. We also have the OAuth protocol to make the delegation access to an API securely. This protocol specifically addresses access to resources authorizations. SAML2 meanwhile is a standard proposed by OASIS dedicated to exchanging authentication and authorization data between security domains.

We offer an identity management system model to analyze the attributes of different accounts of a user and enable it to create another account for all of its various accounts by considering all existing data on the canvas. All these protocols and model management system of digital identity that we offer have something in common: unique identification. 


\section{CONCLUSION AND PERSPECTIVES}

This article is the proposal instead of a model predicting the identity of a user on the Internet. That is to say that a user must, from its attribute data, create a unique account for all accounts on the Internet. We first define the digital identity and we found three main categories of the same: declarative identity, acting identity and calculated identity. Before proposing a solution model and then present the results and discussion we reviewed the different digital identity management systems.

The area of most scalable science today remains computing because it has always tried to solve the problem that his next leave in its wake. Since declarative identities allow well conclude membership from one account to a user, we asked whether the same formula will be applied to the calculated identities. Most users are between $62 \%$ and $70 \%$ of couples of compatible attributes and was taken as the maximum decision threshold. In our sample there is a user who has two accounts but is $22.58 \%$ because the most important attributes are different. Should bring back the threshold to $22.58 \%$ ? Track a user on the web from its attribute data obtained from any site remains an as yet to be illuminated.

\section{REFERENCES}

[1] J. Vincent, Identité numérique en contexte Télécom, 2013

[2] R. Clarke. Identification and authentication fundamentals. Xamax Consultancy Pty Ltd, May, 2004.

[3] Future of identity in the information society - http ://www.fidis.net/. [Accessed March 17 mars 2016]

[4] G.J. Ahn, M. N. Ko and M. Shehab, Portable User-Centric Identity Management

[5]https://fr.wikipedia.org/wiki/Identit\%C3\%A9_num\%C3\% A9rique_(Internet) [Accessed 08 April 2016].

[6] G. Sogliuzzo, Identité / Présence numérique quelles traces laissons-nous sur le web? Professeur documentaliste -8 mars 2013.

[7] W.E. Burr, D.F. Dodson, E.M. Newton, R.A. Perlner, W.T. Polk, S. Gupta, and E.A. Nabbus. Sp 800-63-1. electronic authentication guideline. 2011.

[8] K. Cameron. The laws of identity https://www.identityblog.com/?p=354 [Accessed 10 March 2016]

[9] F. Georges, Les composantes de l'identité dans le web 2.0, une étude sémiotique et statistique.

[10] F. Georges, L'identité numérique dans le web 2.0, Centre de Recherche Images, Cultures et Cognition (CRICC), 2008.

[11] M. Francoise Penta /O. Prompt Manuel Jaffrin, La gestion d'identités: une nécessité sur les campus Numériques
[12] https://fr.wikipedia.org/wiki/DataPortability [Accessed 14 november 2016]

[13] Ph. PREUX, Fouille de données Notes de cours, 26 mai 2011

[14] F. RIOULT, GREYC, Fouille de données orientée motifs, méthodes et usages, Équipe Données-DocumentsLangues CNRS UMR 6072

[15] A. Jøsang and S. Pope. User centric identity management. In AusCERT Asia Pacific Information Technology Security Conference, 2005.

[16] R. Harrison. Lightweight directory access protocol (LDAP): Authentication methods and security mechanisms. Technical report, RFC 4513, June 2006.

[17] T. Dierks and E. Rescorla. The transport layer security (tls) protocol version 1.2, 2008.

[18] J. Camenisch and B. Pfitzmann. Federated identity management. Security, Privacy, and Trust in Modern Data Management, pages 213-238, 2007.

[29] K. Cameron. The laws of identity. Microsoft Corp.

[20] D. Recordon and D. Reed. Openid 2.0 : a platform for user-centric identity management. Page 16, 2006.

[21] R. Fielding, J. Gettys, J. Mogul, H. Frystyk, L. Masinter, P. Leach, and T. Berners-Lee. Hypertext transfer protocolHTTP/1.1, 1999.

[22] E. Rescorla. Http over tls, 2000.

[23] D. Reed and D. McAlpin. Extensible resource identifier (xri) syntax v2. 0.

[24] D. Eastlake and P. Jones. US secure hash algorithm 1 (SHA1), 2001.

[25] E. Hammer-Lahav and D. Recordon. The oauth 1.0 protocol. Internet Engineering Task Force (IETF) RFC5849, pages $2070-1721,2010$.

[26] J. Jonsson et al. Public-Key Cryptography Standards (PKCS) 1: RSA Cryptography Specification Version 2.1. 2003.

[27] S. Cantor, J. Kemp, et al. Liberty ID-FF Protocols and Schema Specification. Version, 183:1-2.

[28] T. Bray, J. Paoli, C.M. Sperberg-McQueen, E. Maler, and F. Yergeau. Extensible markup language (XML) 1.0. W3C recommendation, 6,2000

[29] M. Bartel, J. Boyer, B. Fox, B. LaMacchia, and E. Simon. XML-signature syntax and processing. W3C recommendation, 12: 2002, 2002.

[30] http://www.zdnet.fr/actualites/quand-les-api-vont-tousnous-connecter-39817096.htm [Accessed 23 June 2016] 